\title{
ON COMPACTIFICATION AND STRUCTURE OF TOPOLOGICAL GROUPS
}

\author{
R. T. RAMSAY 1,2
}

The class of locally compact groups $G$ with center $Z$ such that $G / Z$ is compact was investigated by Grosser and Moskowitz [5], [6]. They show that these groups have equal left and right uniformities, that they are maximally almost periodic, and that they are of the form $V \times H$ where $V$ is a vector group, and $H$ has a compact open normal subgroup. It is well known that a group is maximally almost periodic if and only if it has a compactification in the sense that there is a continuous isomorphism of the group into a compact group [2]. For locally compact connected groups $G$, the following are equivalent; (1) $G / Z$ is compact, (2) $G$ has a compactification and (3) $G$ has equal uniformities [14]. In this paper we are primarily interested in finding other classes of groups for which the three properties mentioned above are related. For example, in Theorem 6 , we show that (1), (2) and (3) are equivalent for a certain class of compactly generated groups.

The following lemma is a natural topological version of a group theoretic construction. For a group $G, Z=Z(G)$ is the center of $G$.

Lemma. Let $G_{1}, G_{2}$ be topological groups such that there is a group $H$, and isomorphisms $\phi_{1}, \phi_{2}$ on $H$ such that

(1) $H_{i}=\phi_{i}(H) \subseteq Z\left(G_{i}\right)(i=1,2)$,

(2) $\phi_{2} \phi_{1}^{-1}: H_{1} \rightarrow H_{2}$ is continuous, and

(3) $H_{1}$ is closed in $G_{1}$.

Then $\Delta H=\left\{\left(\phi_{1}(h), \phi_{2}\left(h^{-1}\right)\right): h \in H\right\}$ is a closed normal subgroup of $G_{1} \times G_{2}$, and the natural homomorphism of $G_{i}(i=1,2)$ into $\left(G_{1} \times G_{2}\right) / \Delta H$ is a continuous isomorphism.

Proof. The algebraic aspects of this can be found in $[13$, p. 184]. The topological considerations are straightforward.

In Theorem 1 we do not assume local compactness. Thus we have generalized Theorem 3.1 in [6].

THEOREM 1. If $G$ is a topological group such that $Z$ has a compactification and $G / Z$ is compact, then $G$ has a compactification. 1967.

Presented to the Society, August 31, 1967; received by the editors October 24,

${ }^{1}$ I wish to express my gratitude to R. W. Bagley and T. S. Wu for their help in the preparation of this paper.

2 This work is a part of the author's dissertation. 
Proof. Let $\phi$ be a continuous isomorphism of $Z$ into the compact group $\bar{Z}$. We may assume that $\phi(Z)$ is dense in $\bar{Z}$, and so $\bar{Z}$ is abelian. Thus by our lemma, there is a continuous isomorphism of $G$ into $(G \times \bar{Z}) / \Delta Z$. We need only show that this last group is compact. There are continuous isomorphisms of $\bar{Z}$ onto $(Z \times \bar{Z}) / \Delta Z$ and $G / Z$ onto $(G \times \bar{Z}) /(Z \times \bar{Z})$. Thus $(Z \times \bar{Z}) / \Delta Z$ and $(G \times \bar{Z}) /(Z \times \bar{Z})$ are compact, and it follows that $(G \times \bar{Z}) / \Delta Z$ is compact, since $(G \times \bar{Z}) /(Z \times \bar{Z})$ $\cong[(G \times \bar{Z}) / \Delta Z] /[(Z \times \bar{Z}) / \Delta Z]$.

Corollary. If $G$ is locally compact and $G / Z$ is compact, then $G$ has a compactification.

Proof. Let $\bar{Z}$ be the Bohr compactification of $Z$.

We state the following theorem for completeness. It is Theorem 2.3 of $[5]$.

THEOREM 2. If $G$ is a locally compact group such that $G / Z$ is compact, then $G \cong V \times H$, where $V$ is a vector group and $H$ has a compact open normal subgroup.

Let $G^{\prime}$ denote the closure of the commutator subgroup of $G$. It is a corollary to Theorem 2 that if $G / Z$ is compact, then $G^{\prime}$ is compact [5]. The following structure theorem is thus clearly related to Theorem 2.

THEOREM 3. If $G$ is locally compact, $G^{\prime}$ compact, and $G$ has a closed abelian subgroup $G_{1}$ such that $G / G_{1} G^{\prime}$ is compact, then $G$ is the semidirect product of $H$ by $V$, where $H$ has no nontrivial vector subgroup and $V$ is a vector group.

Proof. Let $G_{1} \cong V \times H_{1}$ where $V$ is a vector group and $H_{1}$ has a compact open subgroup. Since $V$ has no nontrivial compact subgroups, $V \cap G^{\prime}=\{e\}$. Thus $G / H_{1} G^{\prime}$ is an abelian group with a vector subgroup $G_{1} G^{\prime} / H_{1} G^{\prime} \cong V$ such that the factor group $\left(G / H_{1} G^{\prime}\right) / V$ $\cong G / G_{1} G^{\prime}$ is compact. Thus by Lemma 3.8 of [9], we have $G / H_{1} G^{\prime}$ $\cong V \times K$ where $K$ is a compact group. Let $H$ be the inverse image of $K$ in $G$. Then it is clear that $H$ is a normal subgroup of $G$ such that $H V=G$ and $H \cap V=\{e\}$. Finally, $H$ has no nontrivial vector subgroup, since $H / H_{1} G^{\prime} \cong K$ is compact, and $H_{1} G^{\prime}$ has a compact open subgroup. The proof is complete.

For a locally compact group $G$, let $A(G)$ denote the group of all homeomorphic automorphisms of $G$. This group has a topological group topology such that the natural homomorphism of $G$ into $I(G)$, the subgroup of inner automorphisms, is continuous [8, p. 428]. This topology is the smallest admissible topology such that $A(G)$ is a topo- 
logical group [1]. If $G$ has equal uniformities, then $I(G)$ is equicontinuous, and the topology of $A(G)$ restricted to $I(G)$ is just the compact open topology [3]. When $G / Z$ is compact, then $I(G)$ is compact, and hence closed in $A(G)$. Thus it is natural in what follows to consider the class of groups for which $I(G)$ is a closed subgroup. By invariant neighborhood, we mean invariant under the inner automorphisms of $G$.

THEOREM 4. If a group $G$ is generated by a compact invariant neighborhood of $e, I(G)$ is closed, and $G$ has equal uniformities, then $G / Z$ is compact.

Proof. Let $U$ be a compact invariant neighborhood of $e$ such that $G=\cup_{n=1}^{\infty} U^{n}$. If $x \in U^{n}$, then $I(G)[x]=\left\{g x g^{-1}: g \in G\right\} \subseteq U^{n}$, and $U^{n}$ is compact. By an Ascoli theorem, $I(G)$ is compact. Since $G / Z$ is $\sigma$-compact, it follows that the isomorphism from $G / Z$ onto $I(G)$ is a homeomorphism, and so $G / Z$ is compact.

We shall say that a group is compactly generated if it is generated by a compact neighborhood of $e$. Of course this implies that the group is locally compact.

CoROLlary. If $G$ is compactly generated and has equal uniformities, then the following are equivalent:

(1) $G^{\prime}$ is compact and $I(G)$ is closed.

(2) $G / Z$ is compact.

Proof. If $G^{\prime}$ is compact and $U$ is a compact neighborhood of $e$ generating $G$, then $G^{\prime} U$ is a compact invariant neighborhood which obviously generates $G$.

B. H. Neumann proved that if $G$ is a finitely generated group, then $G^{\prime}$ is finite if and only if $G / Z$ is finite [12]. This theorem follows from our corollary by noting that for a finitely generated discrete group, $A(G)$ is discrete $[8$, p. 434], and so $I(G)$ is closed.

We have noted that if $G$ is locally compact and $G^{\prime}$ compact, then there is a compact invariant neighborhood of $e$. The converse is true if $G$ is connected [10]. If $G$ is not connected, we have the following theorem.

THEOREM 5. If $G$ is generated by a compact invariant neighborhood of $e$, and $I(H)$ is closed for each quotient group $H$ of $G$, then $G^{\prime}$ is compact.

Proof. By Theorem 1 in [10], $G$ has a compact normal subgroup $N$ such that $G / N$ has equal uniformities. Thus if $Z$ is the center of $G / N$, then $(G / N) / Z$ is compact by Theorem 4 . Thus $(G / N)^{\prime}$ is compact. Let $K$ be the inverse image of $(G / N)^{\prime}$ in $G$. Then $K$ is a compact 
normal subgroup of $G$ such that $G / K$ is abelian. Thus $G^{\prime} \subset K$, and so $G^{\prime}$ is compact.

Finally, we combine several of our previous theorems to state the following theorem.

Theorem 6. If $G$ is compactly generated, $I(G)$ is closed and $G^{\prime}$ is compact, then the following are equivalent:

(1) G has a compactification.

(2) G has equal uniformities.

(3) $G / Z$ is compact.

Proof. Theorem 1 tells us that (3) implies (1). It is well known that (1) implies (2) [7, p. 49]. Finally, (2) implies (3) by Theorem 4.

We conclude this paper with some questions and remarks. There is a locally compact group which has a compactification, but which fails to have equal uniformities [11], and conversely there is a locally compact group with equal uniformities which fails to have a compactification [8, p. 351]. As a matter of fact, there is a finitely generated discrete group which fails to have a compactification [4, p. 156]. ${ }^{3}$

What conditions on a topological group $G$ imply that $I(G)$ is closed in $A(G)$ ? Several of the theorems in this paper suggest that the answers to this question would be useful.

\section{BIBLIOGRAPHY}

1. R. F. Arens, Topologies for homeomorphism groups, Amer. J. Math. 68 (1946), 593-610.

2. H. Chu, Compactification and duality of topological groups, Trans Amer. Math. Soc. 123 (1966), 310-324.

3. J. Dieudonné, On topological groups of homeomorphisms, Amer. J. Math. 70 (1948), 659-680.

4. W. Fluch, Maximal-Fastperiodizität von Gruppen. I, Math. Scand. 16 (1965), 148-158.

5. S. Grosser and M. Moskowitz, On central topological groups, Bull. Amer. Math. Soc. 72 (1966), 826-830.

6. - Representation theory of central topological groups, Bull. Amer. Math. Soc. 72 (1966), 831-837.

7. K. H. Hofman and P. Mostert, Splitting in topological groups, Mem. Amer. Math. Soc. No. 43 (1963).

8. E. Hewitt and K. A. Ross, Abstract harmonic analysis, Academic Press, New York, 1963.

9. K. Iwasawa, On some types of topological groups, Ann. of Math. 50 (1949), 507-558.

3 The author is indebted to the referee for this example. 
10. - Topological groups with invariant neighborhoods of the identity, Ann. of Math. 54 (1951), 345-348.

11. S. Murakami, Remarks on the structure of maximally almost periodic groups, Osaka J. Math. 2 (1950), 119-129.

12. B. H. Neumann, Groups with finite classes of conjugate subgroups, Math. Z. 63 (1955), 76-96.

13. W. R. Scott, Group theory, Prentice-Hall, Englewood Cliffs, N. J., 1964.

14. A. Weil, L'integration dans les groupes topologiques et ses applications, Hermann, Paris, 1953.

North Carolina State University 